\title{
NUMERI CARATTERISTICI DEI FLOCCULI D'IDROGENO E DI CALCIO E DEI FILAMENTI D'IDROGENO PER L'ANNO 1954
}

\author{
G. GODOLI
}

Proseguendo la pubblicazione annuale dei numeri caratteristici dei flocculi d'idrogeno e di calcio e dei filamenti di idrogeno ('), rengrono riportati nelle seruenti tre tabelle di immediata interpretazione i dati per il 1954.

I numeri caratteristici sono stati stimati sugli spettroeliogrammi eseguiti alla torre solare di Arcetri con l'immagine di $64 \mathrm{~mm}$; le caratteristiche strumentali e la tecnica delle osservazioni sono state descritte in altra sede $1 \%$

Sono state esaminate complessivamente 116 lastre in $H \propto \mathrm{e} 110$ in $K$

La stima reniva eseguita, come per il passato, con l'aiuto dei modelli di Mt. Wilson. Per evidenti ragioni di uniformità è stata determinata prima tutta la serie dei numeri caratteristici per i flocculi d'idrogeno; quindi tutta la serie per i flocculi di calcio ed infine tutta la serie per i filamenti d'idrogeno. Lo scarto medio delle singole determinazioni $i$ stato sempre inferiore a 0.2 unità.

Il contributo dei singoli osservatori nella raccolta delle lastre è stato il seguente:

\begin{tabular}{|l|r|r|r|}
\hline Osservatori & $\mathrm{H} \alpha$ & $\mathrm{K}_{\mathrm{e}, 3}$ & Totale \\
\cline { 2 - 3 } Bullario & 82 & 77 & 159 \\
Gololi & 17 & 16 & 33 \\
Maffei & 3 & 3 & 6 \\
Riguti & 13 & 13 & 26 \\
Romagnoli & 1 & 1 & 2
\end{tabular}

Gli osservatori sono stati sempre coadiurati dal sig. Romagnoli. 
Tabella 1-a

Numeri caratteristici dei flocculi d'idrogeno per lanno 1954

\begin{tabular}{|c|c|c|c|c|c|c|c|c|c|c|c|c|}
\hline$\frac{\stackrel{̊}{5}}{\stackrel{5}{3}}$ & 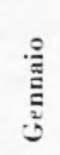 & 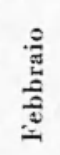 & $\stackrel{\stackrel{i}{*}}{z}$ & 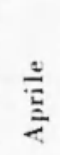 & 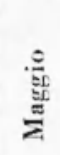 & $\stackrel{0}{3}$ & $\begin{array}{l}\stackrel{0}{ت} \\
\stackrel{80}{\Xi}\end{array}$ & 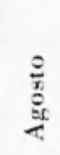 & 气ัّ & $\frac{5}{5}$ & 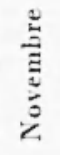 & $\frac{\vdots}{\bar{\Xi}}$ \\
\hline 1 & - & - & - & - & - & - & - & - & 0.0 & 0.1 & - & - \\
\hline 2 & 0.0 & - & - & - & - & - & - & 0.2 & - & 0.2 & 0.0 & - \\
\hline 3 & - & - & - & 0.0 & - & - & 0.1 & 0.2 & 0.0 & - & - & - \\
\hline 4 & - & - & - & - & - & - & - & 0.2 & - & - & - & 0.0 \\
\hline 5 & - & - & - & - & - & - & - & - & - & 0.4 & 0.1 & \\
\hline 6 & - & - & - & - & - & - & 0.0 & 0.4 & 0.2 & 0.4 & 0.1 & - \\
\hline 7 & - & - & - & - & 0.0 & - & - & 0.3 & 0.2 & - & - & - \\
\hline 8 & - & - & 0.0 & 0.1 & - & 0.0 & 0.0 & - & - & - & - & - \\
\hline 9 & - & - & 0.0 & 0.1 & - & - & - & - & - & - & - & - \\
\hline 10 & - & 0.1 & 0.0 & - & 0.0 & - & - & - & - & - & - & - \\
\hline 11 & - & - & 0.0 & - & - & 0.0 & - & -- & 0.1 & - & - & - \\
\hline 12 & - & - & - & 0.4 & 0.0 & 0.0 & - & 0.2 & - & - & - & - \\
\hline 13 & - & 0.1 & - & 0.5 & 0.0 & - & - & 0.2 & 0.0 & - & 0.4 & - \\
\hline 11 & - & - & - & 0.5 & 0.0 & - & 0.1 & - & 0.1 & - & - & - \\
\hline 15 & 0.0 & - & - & 0.5 & - & - & 0.1 & - & - & - & 0.2 & 0.1 \\
\hline 16 & - & - & 0.6 & - & - & - & 0.1 & - & - & - & 0.2 & 0.2 \\
\hline 17 & - & - & - & - & - & - & 0.1 & 0.0 & 0.1 & - & - & 0.2 \\
\hline 18 & 0.0 & 0.0 & - & - & - & 0.0 & - & 0.0 & - & - & - & 0.2 \\
\hline 19 & - & 0.0 & - & - & - & - & 0.1 & 0.0 & - & - & 0.1 & - \\
\hline 20 & - & - & - & - & - & - & 0.1 & 0.0 & - & - & 0.1 & 0.1 \\
\hline 21 & - & - & - & - & 0.0 & - & 0.0 & - & 0.1 & - & - & - \\
\hline 22 & - & - & 0.2 & 0.0 & - & 0.0 & 0.0 & - & 0.0 & - & 0.0 & - \\
\hline 23 & 0.0 & 0.0 & 0.1 & - & - & 0.0 & 0.0 & - & 0.1 & - & 0.0 & - \\
\hline 24 & - & 0.0 & - & - & - & - & 0.1 & - & 0.0 & - & - & - \\
\hline 25 & 0.1 & - & - & - & 0.0 & - & - & - & 0.2 & - & - & - \\
\hline 26 & - & - & - & - & - & 0.0 & 0.3 & - & - & - & - & - \\
\hline 27 & - & - & - & - & - & - & 0.3 & - & - & 00 & - & - \\
\hline 28 & 0.1 & - & - & - & - & 0.0 & 0.3 & 0.0 & - & 0.0 & - & - \\
\hline 29 & - & - & 0.0 & - & - & - & 0.3 & - & - & 0.0 & - & - \\
\hline 30 & - & - & 0.0 & - & - & - & 0.3 & 0.0 & 0.1 & 0.0 & - & 0.1 \\
\hline 31 & - & - & 0.0 & - & - & - & - & 0.0 & - & - & - & - \\
\hline Media & 0.0 & 0.0 & 0.1 & 0.3 & 0.0 & 0.0 & 0.1 & 0.1 & 0.1 & 0.1 & 0.1 & 0.1 \\
\hline
\end{tabular}




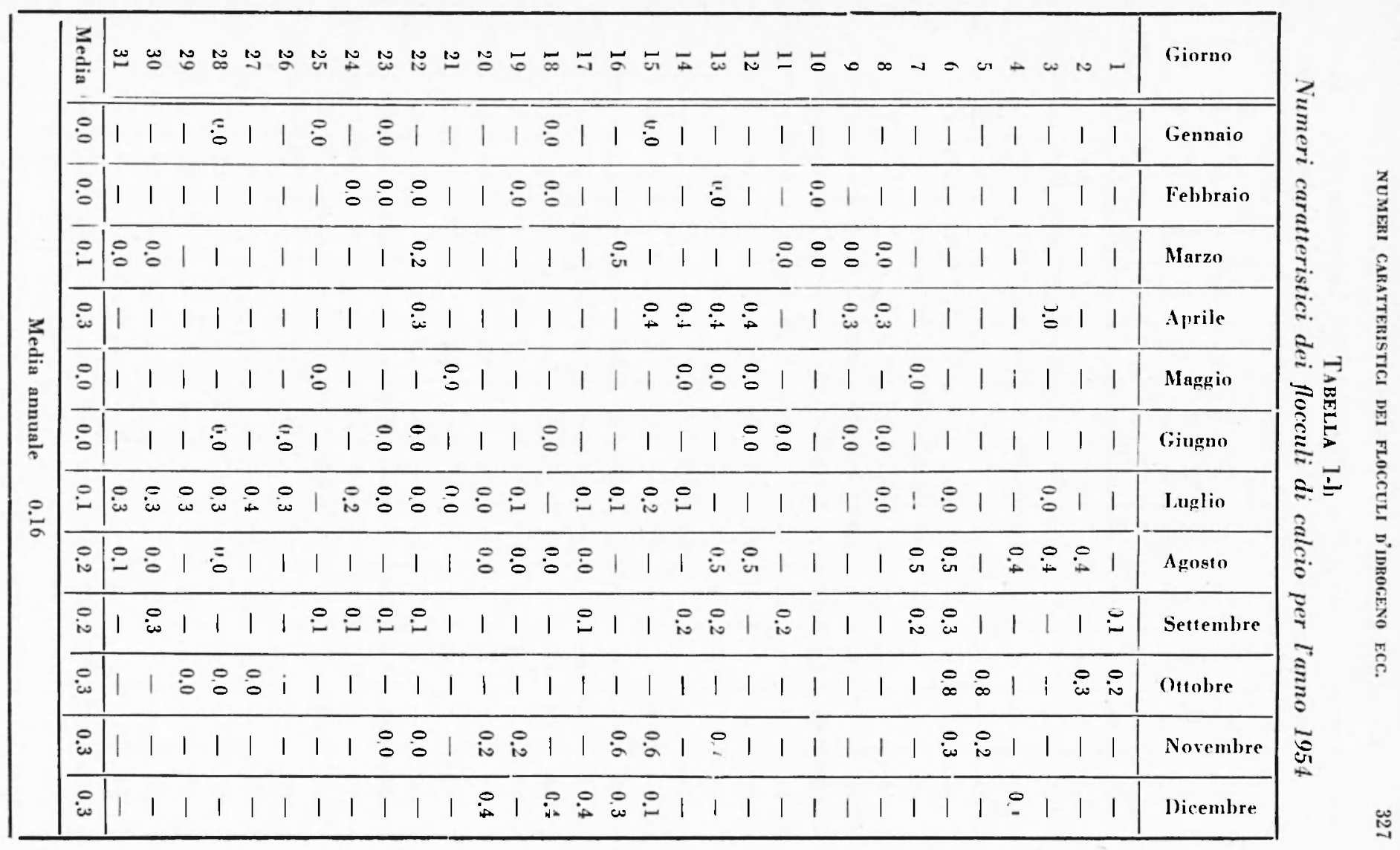




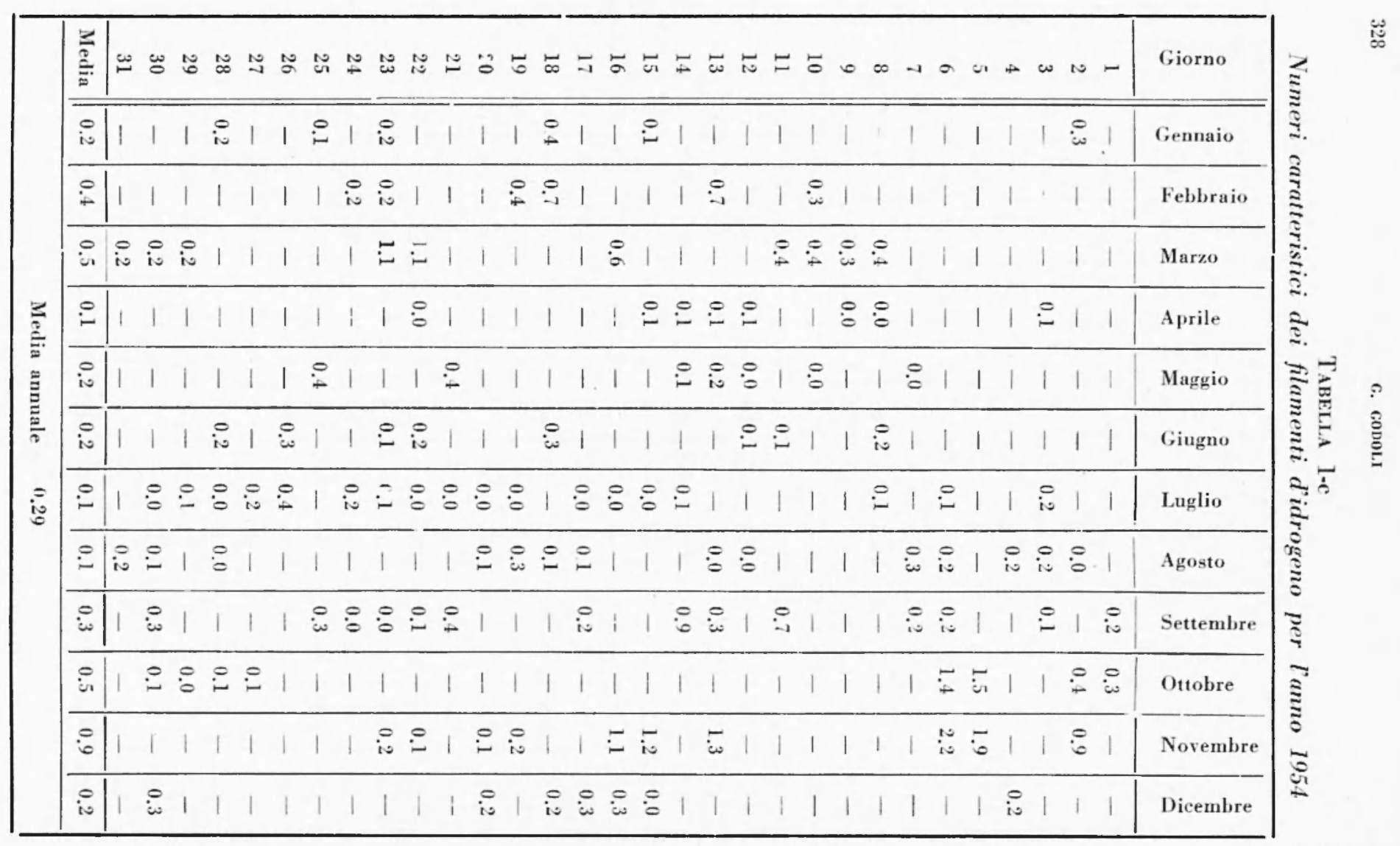


La distribuzione mensile delle lastre ridotte ̀̀ qui sotto indicata:

\begin{tabular}{|l|c|c|c|c|c|c|c|c|c|c|c|c|}
\hline & 1 & 2 & 3 & 4 & 5 & 6 & 7 & 8 & 9 & 10 & 11 & 12 \\
\hline $\mathrm{H} \%$ & 6 & 6 & 10 & 8 & 7 & 8 & 18 & 14 & 14 & 8 & 10 & 7 \\
\hline $\mathrm{K}_{-,: 5}$ & 5 & 7 & 8 & 8 & 6 & 9 & 19 & 14 & 12 & 7 & 9 & 6 \\
\hline
\end{tabular}

Firenze - Osservatorio astrofisico di Arcetri - Centro di astrofisica del C. N. R. - Gennaio 1955.

\section{RIASSUNTO}

Si dànno i numeri caratteristici dei flocculi didrogeno e di calcio e dei filamenti didrogeno per lanno 1954, secondo le osservazioni eseguite alla torre solare di Arcetri.

\section{$S L M M A R Y$}

The character figures of $\mathrm{Ca}$ flocculi and of $\mathrm{H}_{\alpha}$. bright and dark flocculi for the year 1954 are given according to the observations carried out at the solar touer of Arcetri.

\section{BIBLIOGRAFIA}

(1) G. Gonol., Numeri caratteristici dei focculi didrogeno e di calcio e dei filamenti didrogeno per gli anni 1950.51.52, Ann. Geof. 6. 199. (1953).

G. Gobou, Numeri caratteristici dei flocculi didrogeno e di calcio e dei fillamenti didrogeno per lanno 1953, Ann. Geof. i, 135. 11954$).$

(2) G. Righivi e G. Godoli, Riduzione del materiale spettroeliografico raccolto alla torre solare di Arcetri nel periodo 1932.1949. Mem. SAIt. 21. 333. 1950). 